Detection and discrimination of colour, a comparison of physiological and psychophysical data

This content has been downloaded from IOPscience. Please scroll down to see the full text.

1989 Phys. Scr. 39178

(http://iopscience.iop.org/1402-4896/39/1/029)

View the table of contents for this issue, or go to the journal homepage for more

Download details:

IP Address: 134.76.223.157

This content was downloaded on 02/03/2017 at 13:17

Please note that terms and conditions apply.

You may also be interested in:

Colour representation spaces at different physiological levels: a comparative analysis

P Capilla, J Malo, M J Luque et al.

Ideal observer theory in psychophysics and physiology

Wilson S Geisler

The organization of human vision for pattern detection and recognition

K H Ruddock

Current problems of visual research

W S Stiles

Modern Problems of Colorimetry

W D Wright

Sense organs - transducers of the environment

C Rashbass

Colour reproduction by photography

R W G Hunt

The influence of chromatic adaptation upon successive colour discrimination

E Hita, J Romero, A Cervantes et al.

Colour memory matching analysed using different representation spaces

M D de Fez, M J Luque, P Capilla et al. 


\title{
Detection and Discrimination of Colour, a Comparison of Physiological and Psychophysical Data
}

\author{
A. Valberg* and B. B. Lee \\ Institute of Physics, Dept. of Biophysics, University of Oslo, Oslo, Norway and Max Planck Institute for Biophysical Chemistry, Goettingen, \\ West-Germany
}

Received February 1, 1987: accepted February 1, 1988

\begin{abstract}
Whereas the physiological basis of colorimetry (colour matches) is well understood in terms of the trireceptor theory of colour vision, colour discrimination and scaling still lack a comparable foundation. We present here experimental data that demonstrate how sensitivity and responsiveness of different types of cone-opponent and non-opponent cells of the macaque monkey correlate with human threshold sensitivity on the one hand, and how they in combination can be used to construct a suprathreshold equidistant colour space. Psychophysical thresholds correlate well with the threshold envelope of the most sensitive cells when stimuli are projected upon a steady white background. Detection thresholds for stimuli of differing wavelength and purity (saturation) generally indicate a transition from a phasic nonopponent system to a tonic opponent system of on-centre cells as purity increases. Detection and chromatic discrimination thresholds coincide only for long and short wavelengths of high purity, whereas they differ for mid-spectral lights. Different cell types may thus support detection and discrimination with different stimuli. With chromatic scaling of surface colours on the other hand, when stimuli are darker than an adaptation field still other cell types are needed. We demonstrate that it is possible, from a combination of on- and off-opponent cells, to reconstruct a uniform colour space, using summed outputs of cells with the same cone combination and vector addition for cells with different combinations. Different hues are represented by opponent cells with inputs from different cone types, the hue percept being related to the ratio of the activities of these cell systems.
\end{abstract}

\section{Introduction}

Physical colour measurement, or colorimetry, is based on the visual match between some unknown light and an additive mixture of three standard lights, usually red, green, and blue. The results of colour-matching experiments can be fully understood in terms of the trichromatic theory of colour vision, where the three cones types can be asssumed to behave like linear physical detectors (Schroedinger's "niedere Farbmetrik" [1]). Matching experiments tell us nothing about the sensitivity, gain, etc. of these detectors or other underlying mechanisms. Such information can be obtained by determining detection and discrimination thresholds. Usually, in detection experiments, judgements of qualitative attributes of the stimuli are irrelevant, and the eye can again be treated as a physical instrument. The determination of threshold sensitivities is a common approach in vision for it allows direct comparison of psychophysical and physiological data.

In order to establish an equidistant colour metric (Schroedinger's "hoehere Farbmetrik" [1]), we must turn to colour discrimination and scaling. One then asks "which pairs of light stimuli will evoke the same sensory colour differences"? Is it possible to derive a scale, that can be used

\footnotetext{
* Address for correspondence: A. Valberg, Institute of Physics, Dept. of Biophysics, University of Oslo, Box 1048 Blindern, N-0316 Oslo 3, Norway.
}

in colour space such that a fixed distance in this space represents the same colour difference throughout, regardless of the lightness and colour of the pair, whether light or dark, reddish or greenish? With such questions linear colour metrics fail, and a more sophisticated approach is necessary, perhaps due to the non-linearity of physiological responses themselves. A related question is that of the qualitative attributes of light and colour and their possible cellular representation.

The integration of these three stages, the physical, physiological, and perceptual, into an unified colour vision model has proved difficult. The first attempts to account for human colour difference sensitivity and scaling were in terms of line elements in three-dimensional vector- or cone space, a direct extension of trichromatic theory. Famous physicists such as Helmholtz [2], Schroedinger [1], and Stiles [3] were associated with this approach. However, such treatments have not been particularly successful, and neither have been the many other attempts to describe suprathreshold discrimination within the framework of trichromatic theory alone.

It thus seems desirable to investigate alternative ways of analysing human detection and discrimination performance. A promising approach is the measurement of physiological sensitivity and responses in the macaque monkey to visual stimuli, not only at the receptor level, but also of neurones in the retina and higher visual centres. Transformation of the receptor signals into a more efficient code of spectral opponency and/or spatial antagonism takes place in these cells' receptive fields; the visual system of the macaque is very similar to that of man, both in terms of spectral absorption of the receptors $[4,5]$ and in terms of anatomy of the visual pathway. Psychophysically the two species are also similar [6]. In the retina of the macaque, and most probably in man, there exist tonic, wavelength-opponent ganglion cells which project to the parvocellular layers (PCL) of the lateral geniculate nucleus (LGN), and phasic, non-opponent cells which project to the magnocellular layers (MCL) of the LGN [7-12].

In relating physiological responses to psychophysical data there are several pitfalls that must be avoided. For instance, it is important to differentiate between different stimulus conditions, for example, the relationship of a stimulus to its surroundings. We have obtained strong evidence that the detection of incremental spots on a dark or a steady white background is based on cell types other than those which are the most sensitive to object colours ("related colours" $[13,14])$ for which luminance factors (stimulus/surround luminance ratios) range between zero (black) and one (white). Cells of different types may thus underlie detection and 
scaling under the differing stimulus conditions. Therefore, generalisations about the mechanisms subserving detection and discrimination from one set of stimulus conditions to another are usually invalid.

Here we compare cell outputs with psychophysical data from the literature and with data we have obtained using the same stimulus conditions as in the recordings from the visual pathway of the macaque monkey. Our aim was to establish the relationship between cell sensitivity and response on the one hand, and psychophysical discrimination on the other, and so deduce possible roles played by different cell types in detection and suprathreshold discrimination tasks.

\section{Methods}

The neurophysiological material has been taken from an extensive study of various aspects of colour coding in the LGN of Macaca fascicularis [13-18]. In 24 monkeys which were lightly anesthetised with a nitrous oxide/oxygen mixture with the addtion of $0.2-0.6 \%$ halothane, cell activity was recorded through a microelectrode lowered into the LGN. Passage of the microelectrode from PCL into MCL is accompanied by characteristic changes in cell response properties. Receptive field eccentricities were typically about $10^{\circ}$ parafoveal; a total of 567 neurones were studied.

Stimuli were back-projected onto a translucent screen $57 \mathrm{~cm}$ in front of the animal's eyes. Stimuli and backgrounds were generated with a threee-channel optical system, one channel providing a steady background or an alternating adaptation field, the other two various stimuli configurations. The light sources for each channel were Prado Universal projectors (Leitz), providing white light with CIE chromaticity coordinates $(x, y)=(0.404,0.410)$. The adaptation field and the steady background had a luminance of $110 \mathrm{~cd} / \mathrm{m}^{2}$, corresponding to $3100 \mathrm{td}$ with a $6 \mathrm{~mm}$ diameter artificial pupil.

Responses were measured either to spots $\left(4^{\circ}\right.$ or $\left.0.5^{\circ}\right)$ flashed ( $300 \mathrm{~ms}$ duration) on the white backgroumd or in a successive contrast paradigm where large $\left(4^{\circ} \times 5^{\circ}\right)$ chromatic and achromatic stimuli ( $300 \mathrm{~ms}$ duration) were alternated with a white adaptation field (1.2s duration). Some experiments were done with and some without a white surround around the central stimuli [15]. Chromatic stimuli were generated by interference filters (Schott NAL). Responses to such stimuli (the average of 5 or 10 stimulus repetitions) were plotted as a function of luminance increment $\Delta L / L$ (for steady background), or as a function of increasing luminance ratio $\left(Y=L / L_{\mathrm{a}}\right), L$ denoting stimulus luminance and $L_{\mathrm{a}}$ adaptation field luminance (for the successive contrast paradigm). Luminance was computed from the spectral distribution of the stimuli on the screen by using the CIE $10^{\circ} V_{\lambda}$ curve. Computed values were checked by a Spectrophotometer (702A/703A from Photo Research). A more detailed description of preparation and procedures is found elsewhere $[14,17,18]$.

To measure cell thresholds, we constructed luminanceresponse curves for different wavelengths using motor-driven wheels holding 10 neutral-density filters (in steps of 0.2 or 0.3 $\log$ unit) to give ascending series of stimulus intensities. To measure responses, for transient MCL cells firing rate in the pcak 3 bins $(45 \mathrm{~ms})$ of the response was calculated, and for PCL cells the mean firing rate over the entire response. After subtraction of maintained activity to the white background (or alternating field), luminance-response curves were plotted and thresholds defined as a $10 \mathrm{impulse} / \mathrm{s}$ increment in firing rate, as illustrated in the results section.

In psychophysical experiments where human subjects determined detection and chromatic thresholds on a white background, the filter wheels held neutral-density filters covering a $1 \log$ unit range in $0.1 \log$ unit steps but in random order. Overall luminance was adjusted with filters elsewhere in the beam so that this range straddled threshold. Frequency-of-seeing curves were constructed, and the $50 \%$ level taken as detection threshold. False positives were very rare. Three naive subjects with normal colour vision were used. Thresholds were reproducible within and between observers to within about $\pm 0.1 \log$ unit or less. Chromatic discrimination thresholds were measured with a two-alternative forced-choice procedure. A chromatic and a white spot, normalised to their respective thresholds, were presented in random order, and subjects required to identify the coloured one. Initially below detection threshold, luminance was increased in $0.1 \log$ unit steps until a chromatic discrimination threshold was reached.

\section{Results}

\subsection{Thresholds on a constant white background}

We have recorded from cells of the monkey LGN under a variety of stimulus conditions. For comparison with a psychophysical threshold task, we chose to present chromatic spots upon a white background. Since psychophysically sensitivity to such spots peak at several wavelengths, it has been used to adduce the existence of chromatic channels in the human visual system $[19,20]$.

3.1.1. Cell sensitivity. With a steady white background of $110 \mathrm{~cd} / \mathrm{m}^{2}$, we flashed spots of increasing luminance upon neuronal receptive fields. Responses to such series, typical of those obtained from PCL and MCL cells are shown in the histograms of Figs. 1 and 2. In Fig. 1A are shown the responses of a blue on-centre cell to a $4^{\circ}$ stimulus of dominant wavelength $463 \mathrm{~nm}$, and similar histograms for a green oncentre cell and a red off-centre cell stimulated by $500 \mathrm{~nm}$. With weak stimuli, no response is present but with increasing luminance, responses are evoked which eventually saturate and then decrease.

Figures 2A, B display similar histograms for an on- and an off-centre MCL $\left(0.5^{\circ}\right.$ spot size), respectively. The very transient responses of these cells contrast with the sustained PCL cell discharge.

In Figs. $1 \mathrm{~B}$ and 2C, $\mathrm{D}$ the mean firing rates, during the whole response for PCL cells and during the $45 \mathrm{~ms}$ peak for MCL cells, are plotted as functions of luminance increments. A firing rate increment of $10 \mathrm{impulses} / \mathrm{s}$ was chosen as threshold, read off where luminance-response curves intersect with the dashed lines. For the PCL cells shown, contrast threshold is clearly strongly wavelength-dependent, with the highest incremental sensitivity with short wavelength stimuli, the blue on-centre cell being about 1 log unit more sensitive at $460 \mathrm{~nm}$ than the green on-centre cell which is again about 1 $\log$ unit more sensitive than the red off-centre cell with the same M-L cone input. Obviously, off-centre cells were so strongly suppressed by the white background that a much stronger stimulus was necessary to activate them, although for some off-centre cells, sensitivity approached that of 
A
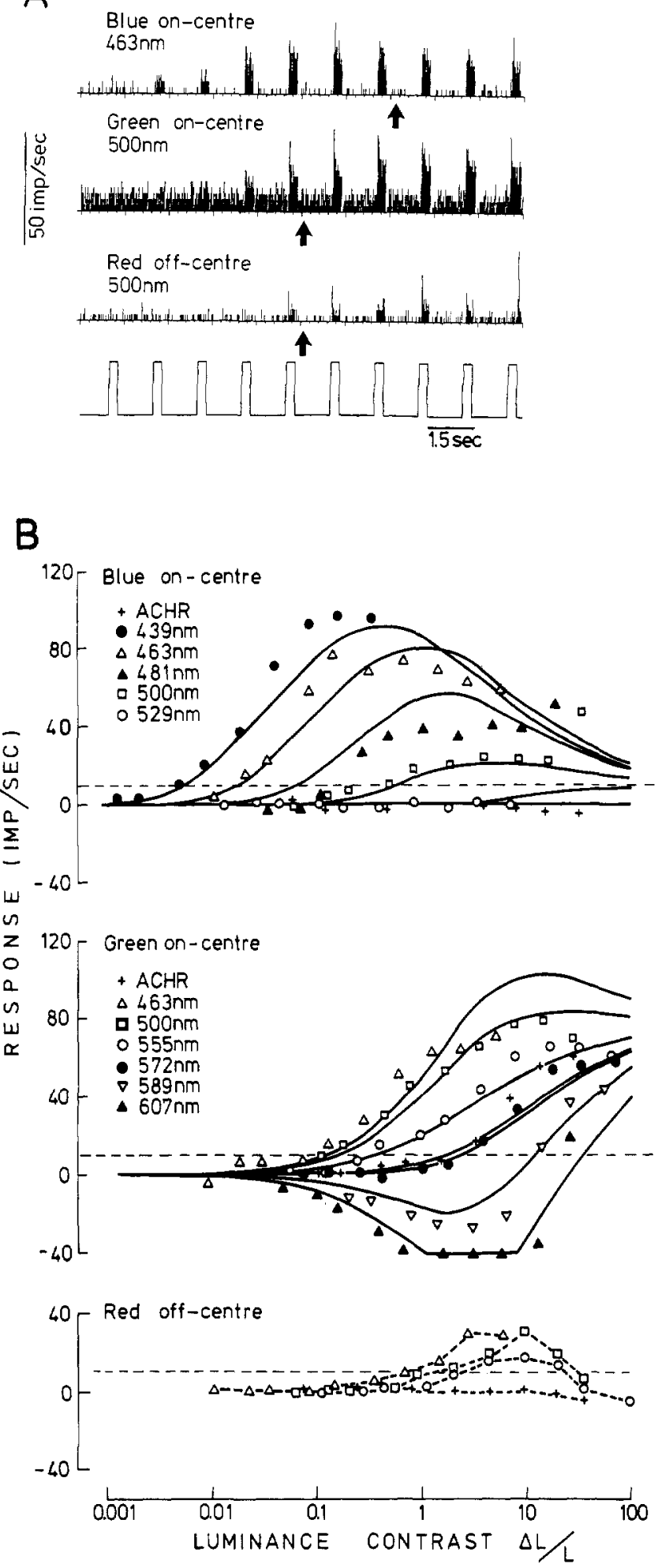

Fig. 1. (A) Histograms of luminance-increment responses of a sustained PCL blue on-centre cell ( $463 \mathrm{~nm}$ stimulus), a green on-centre cell $(500 \mathrm{~nm}$ stimulus), and a red off-centre cell ( $500 \mathrm{~nm}$ stimulus) to $4^{\circ}$ spots superimposed on a steady white, $110 \mathrm{~cd} / \mathrm{m}^{2}$ background. In each run 10 stimuli were presented, increasing in luminance in $0.3 \mathrm{log}$ unit step. Arrows indicate the same luminance contrast of $\Delta L / L=1.0$. For the red off-centre cell, on-responses gradually change to off-responses as luminance contrast increases. (B) Sustained responses plotted as a function of contrast for different wavelengths for the same cells as in (A) (maintained activity subtracted). Threshold and response is highly dependent on wavelength for these PCL cells. The threshold criterion of 10 impulses/s is indicated by the horizontal dashed lines. For the same wavelengths (same symbols), the contrast sensitivity of the red off-centre cell is about $1 \log$ unit less than that for the green on-centre cell (with the same M-L cone combination). The solid curves are those predicted from a model of cone combinations and response behaviour (see text). No simulation was made for the red off-centre cell. on-centre cells. Off-responses were always less sensitive than on-responses.

For the MCL cells in Fig. 2, contrast sensitivity is independent of wavelength, as expected if the spectral sensitivity of phasic ganglion cells is similar to the human $10^{\circ}$ photopic luminosity function $[6,18,21]$. For MCL cells, $4^{\circ}$ spots gave smaller response amplitudes than $0.5^{\circ}$ spots, but with a slightly increased threshold sensitivity.

3.1.2. Simulating cell responses. The curves drawn in Fig. 1B were obtained with a mathematical model that simulates a cell's response, $N$, assuming that the cone inputs to the cell each follow a hyperbolic function as luminance increases, and that spectral opponency is due to linear combinations of signals from different cone mechanisms [17]. A cone mechanism is supposed to be a spatially integrated pool of cone input over the cell's receptive field.

$N=A_{\mathrm{r}} V_{\mathrm{r}}+A_{\mathrm{g}} V_{\mathrm{g}}+A_{\mathrm{b}} V_{\mathrm{b}}+f_{0}$

where

$V_{i}=S_{i}^{0.7} /\left(S_{i}^{0.7}+\sigma_{1}^{0.7}\right), \quad i=r, g, b$

$S_{i}$ is the relative absorption by the receptors, given by $S=$ $\Sigma s_{i} P_{\lambda} \Delta \lambda$, where $s_{i}$ is Estevez's relative spectral sensitivity of a receptor type [22], and $P_{j}$ is the relative power distribution of the stimulus. Here $S$ is equal to the absorption due to the white background, plus that due to the spectral light. The constant $\sigma$ is the absorption giving a half-maximum response, and $V$ is the response of a receptor mechanism, normalised to the maximum. The amplitude $\mathrm{A}$ of a receptor mechanism may enter this equation with a positive (excitation) or a negative (suppression) sign, or be zero. $f_{0}$ is the response to removal of stimulation ("dark response") and may be measured experimentally or derived from the model. Thus there are two free parameters for each cone mechanism ( $A$ and $\sigma$ ), and since the majority of cells have input only from two cone types, a total of four free parameters may describe cell responsiveness over a $5 \mathrm{log}$ unit range of luminance. A more detailed account of the model has been given elsewhere [17].

This model has been used to predict the responses of the blue and green on-centre cells of Fig. 1, and Fig. 1B compare data with the predictions. The agreement is good although deviations are present at higher response magnitudes. The total r.m.s. -deviation for all data points was betwen 7 and 8 impulses/s. For the blue on-centre cell the best simulation was obtained assuming excitatiory S-cone and inhibitory L-cone inputs, with no contribution from M-cones (S-L). The green on-centre cell has $\mathrm{M}-\mathrm{L}$ cone inputs. No simulation was carried out for the red off-centre cell. For the transient MCL cells of Fig. 2 the responsiveness is largely wavelengthindependent. These responses have not been simulated, and we have drawn a common smoothed curve through the data points. Contrast sensitivity at different wavelengths for these cells and for other PCL cells derived as in Figs. 1 and 2, are shown in Fig. 3.

In Fig. 3 we show the contrast sensitivity with spots of different wavelengths of four PCL opponent cells which were among the more sensitive examples when spots were flashed on a steady white background. The curves are derived from the model described above. Below about $480 \mathrm{~nm}$, blue oncentre cells were the most sensitive. Between 480 and $560 \mathrm{~nm}$, green on-centre cells were most sensitive, whereas above 
A
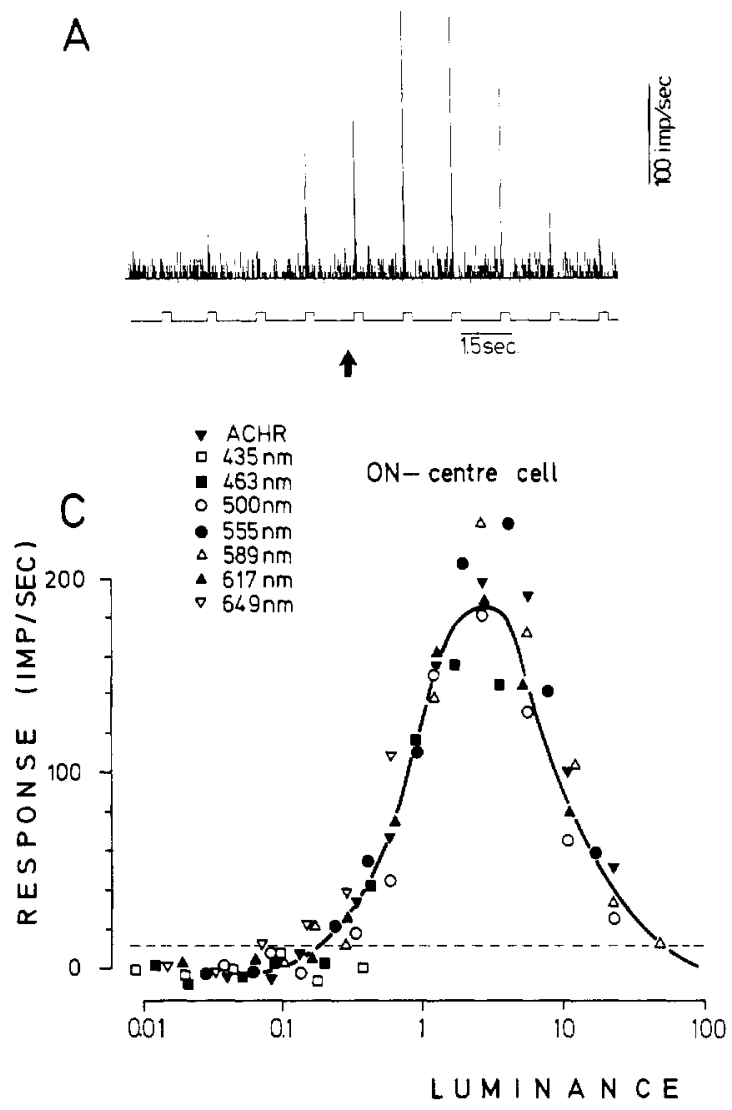

B
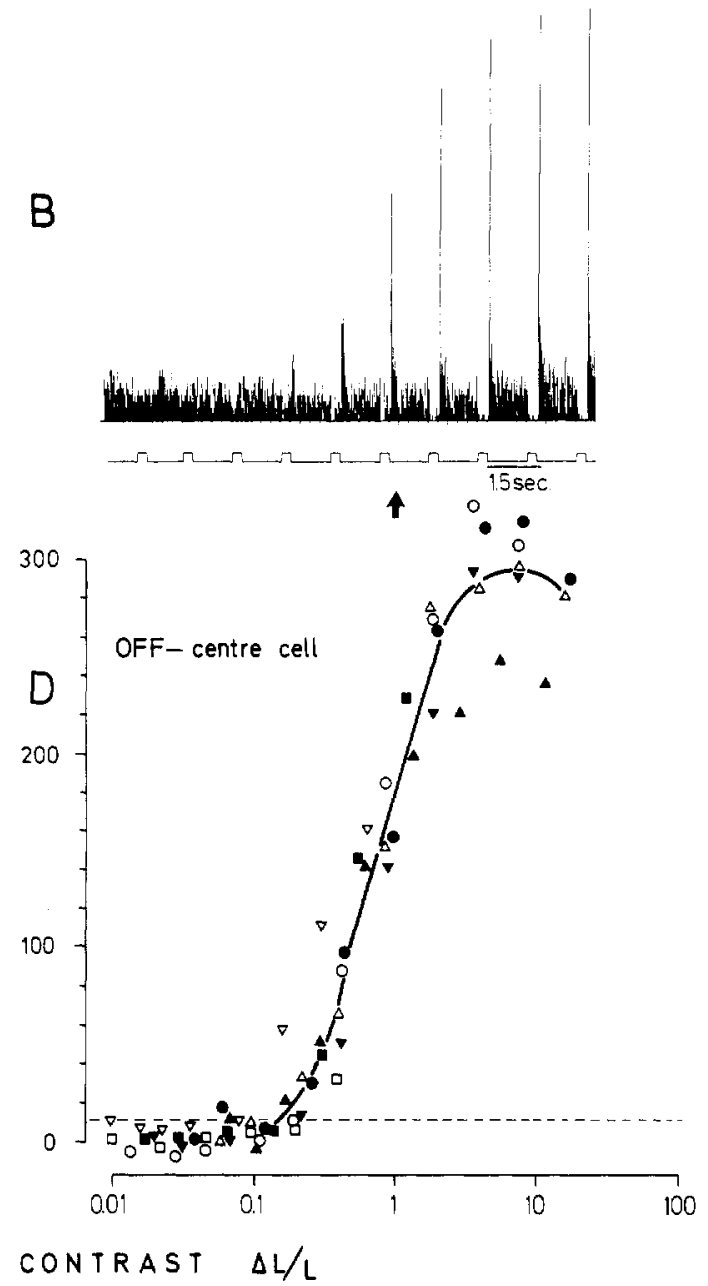

Fig. 2. (A) and (B) Histograms as in Fig. 1(A), but for a transient MCL on-centre and an off-centre cell and $0.5^{\circ}$, $589 \mathrm{~nm}$ spots. (C) and (D) Transient responses (first $45 \mathrm{~ms}$ ) plotted as a function of contrast for different wavelengths for the cells in (A) and (B). Contrary to sustained cells, responses and thresholds are nearly wavelength-independent.

$580 \mathrm{~nm}$, red on-centre cells were most sensitive. Around $570 \mathrm{~nm}$ cells with excitatory M-cone inputs and inhibitory S-cones (M-S) sometimes exceeded the sensitivity of $\mathrm{M} / \mathrm{L}$ cone opponent cells, but $\mathrm{M}-\mathrm{S}$ cells were rare, only constituting $3.5 \%$ of our cell sample [16]. Because the computed curves

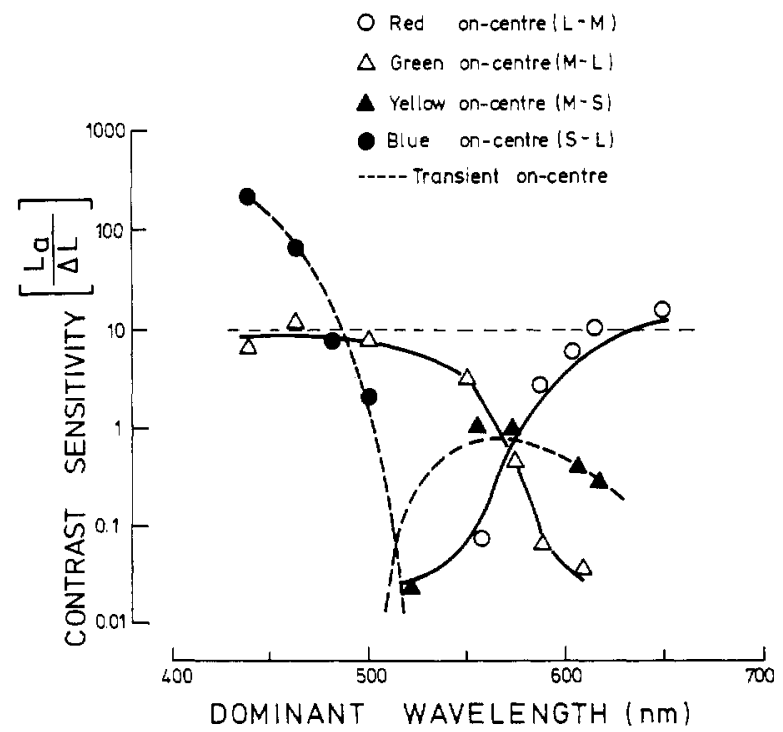

Fig. 3. Spectral contrast sensitivity of four PCL cone-opponent on-cells and one MCL on-cell. The blue- and green on-centre cells are those of Fig. 1. The curves are those derived from the response-model. The horizontal dashed line represent a transient MCL cell. of Figs. 1 and 3 show good agreement with the empirical data, we conclude that it is possible to use the model to determine which cone types feed a particular cell, as well as to estimate the relative sensitivity and strength of these cone mechanisms. This latter information is lost when the usual method of chromatic adaptation is used to reveal cone inputs.

Also plotted in Fig. 3 is the contrast sensitivity of a transient on-centre MCL cell to the same stimulus size. The contrast sensitivity of MCL cells exceeds that of PCL cells by about $1 \log$ unit for the achromatic and $572 \mathrm{~nm}$ stimuli, whereas blue on-centre cells and red on-centre cells are the most sensitive at the spectral ends. The particular cells used in the plots of Fig. 3 are perhaps about $0.2-0.3 \log$ unit less sensitive than the most sensitive cells we found, but their balance and relative spectral sensitivities are representative of the cell population.

3.1.3. Comparison of psychophysical- and cell sensitivities. The sensitivity envelope of the four opponent PCL cells in Fig. 3 resembles psychophysical chromatic discrimination sensitivity [23, 24] for spots on a white background. Several studies have shown that detection and chromatic discrimination thresholds are similar at the spectral ends $[18,20$, 23, 24], where PCL cells have greater sensitivity than MCL cells, whereas for mid-spectral lights there is a difference between luminance increment threshold for detection and for identifification of colour. A possible physiological basis for 


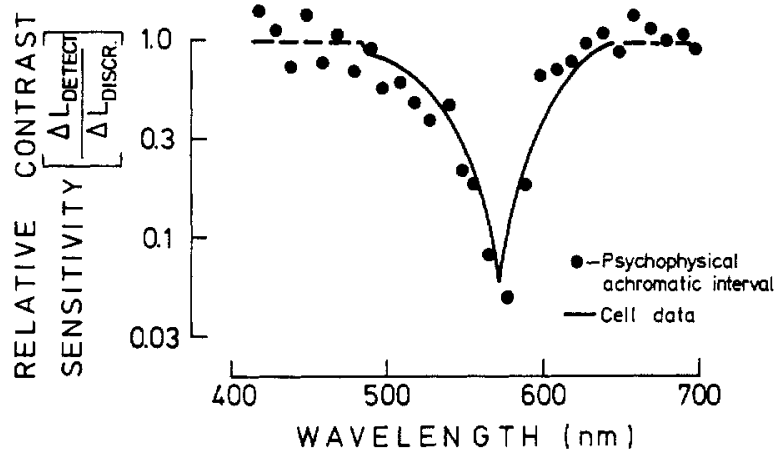

Fig. 4. The solid curve represents the difference between MCL contrast sensitivity and the envelope of PCL cell sensitivities (model curves of Fig. 3) in the wavelength range where transient MCL cells would detect the stimuli before the sustained PCL cells, and where we therefore would expect psychophysical detection thresholds to be lower than chromatic discrimination thresholds. The dashed parts of the curve indicate the spectral regions where PCL cells are more sensitive than MCL cells, and where we would predict detection and discrimination thresholds to be the same. The data points represent the human foveal achromatic interval between detection and colour identification as determined by Graham and Hsia [24].

this result for mid-spectral stimuli, is that detection is mediated by the MCL cells and chromatic discrimination by the PCL cells. At the spectral ends, where PCL cells are most sensitive, they alone could mediate both detection and discrimination. A consequence of this idea is that the achromatic interval betwen detection and identification, as determined for instance by Abney [23] and Graham and Hsia [24], should correspond to the difference in sensitivity of MCL and PCL cells. This is so as demonstrated in Fig. 4, where the MCL and PCL difference of Fig. 3 is compared with the data for Graham and Hsia's [24] foveal achromatic interval (average of 7 observers) on a dark background. Despite the difference in background luminance, the similarity in magnitude and shapes of the curves supports the conjecture.

To provide a direct comparison with our physiological results, for a white background, we also have determined human psychophysical thresholds for detection and chromatic discrimination of $4^{\circ}$ spots at $10^{\circ}$ eccentricity, using the same stimuli as for our neurophysiological recordings. We also found [18] the achromatic interval to be greatest around $570 \mathrm{~nm}$, although of smaller magnitude ( $0.3 \mathrm{log}$ unit) than in Fig. 4. Elsewhere in the spectrum, as in Fig. 4, detection and identification thresholds were the same, that is at the spectral ends below $460 \mathrm{~nm}$ and above $620 \mathrm{~nm}$. With smaller spots $\left(0.5^{\circ}\right)$, cell thresholds also approached psychophysical levels. Our psychophysical results were qualitatively similar to those of King-Smith and Carden [20] for similar stimulus conditions but foveal stimulus presentation.

If MCL cells underlie detection of white spots, and PCL cells of monochromatic spots (except for mid-spectral stimuli), then stimuli of different purities (saturation) may be detected by different systems. For instance, if with a $649 \mathrm{~nm}$ spot, red on-centre cells are the most sensitive, then as purity is decreased by adding white to the stimulus, at some point red on-centre cells would become less contrast sensitive than MCL cells. Up to this point, detection and chromatic discrimination thresholds would be identical, but thereafter chromatic discrimination would require a suprathreshold stimulus.

The continuous lines in Figs. 5A, B show the average contrast sensitivity of two green on-centre cells (Fig. 5A), and

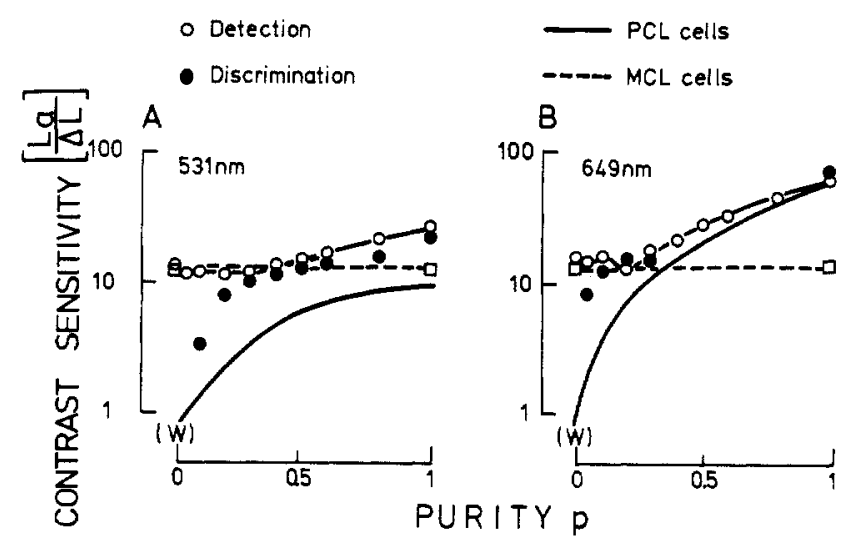

Fig. 5. Comparison of cell thresholds (continuous curves: (A). average of two green on-centre cells. B. average of two red on-centre cells. Horizontal dashed curve: MCL cells) with psychophysical detection and chromatic discrimination thresholds for $4^{\circ}$ spots at $10^{\circ}$ eccentricity. Discrimination and detection sensitivities are similar down to low purities where discrimination sensitivity begins to fall more rapidly like that of the PCL cells, whereas detection sensitivity seems to reach an assymptote at the level of MCL cell sensitivity.

two red on-centre cells (Fig. 5B), plotted as a function of stimulus purity. Purity is defined as $p=L_{\lambda} /\left(L_{\lambda}+L_{\mathrm{w}}\right)$, where $L_{\lambda}$ is the luminance of the chromatic component and $L_{\mathrm{w}}$ the luminance of the white light in the stimulus. For these cells sensitivity increases with increasing purity to reach a sensitivity to chromatic spots 1 or $2 \log$ unit higher than to the white stimulus.

In the same figures we have plotted the threshold sensitivity of human subjects (average of three subjects) to the same stimuli. Both detection and discrimination thresholds were measured. Starting with white light, detection sensitivity stayed constant for low purities $p$ up to $0.2-0.4$ and then increased. Discrimination sensitivity was lower than detection sensitivity for low purities, increasing rapidly as purity increased until at purities of $0.2-0.4$ detection and discrimination thresholds are identical. Opponent cell sensitivity and chromatic discrimination sensitivity follow parallel curves for both wavelengths although the most sensitive cell was for both wavelengths less sensitive than the human subjects. The open squares connected by a dashed line indicate MCL sensitivity, which is expected to be independent of purity and which corresponds to the detection threshold assymptote for low purities.

The experiments displayed in Fig. 5 provide additional support for the hypothesis that the most sensitive mechanism available can provide a basis for detection, and under conditions where MCL cells are the most sensitive, discrimination and detection thresholds are different, PCL cells underlying the discrimination task.

\subsection{Successive contrast. Responses to suprathreshold stimulation}

Although, as shown in the preceding sections, the most sensitive cells may mediate detection in threshold tasks, under suprathreshold conditions a variety of cell types are active and some combination of their outputs must determine the colour perceived. We have recorded suprathreshold responses of opponent cells with a successive contrast stimulus paradigm in an attempt to relate their activities to perceived colour under such conditions.

Under normal viewing conditions, when the eye moves, 


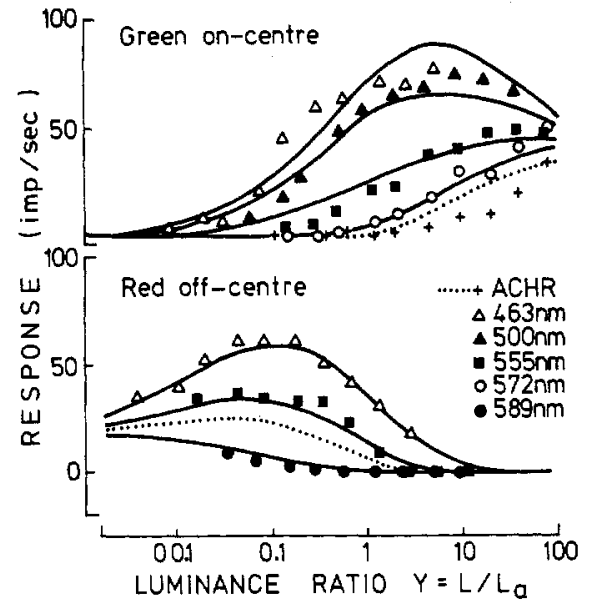

Fig. 6. Examples of luminance-responses of a green on-centre cell and a red off-centre cell when stimuli are alternated with a white adaptation field. Like the cells of Fig. 1, both types receive excitatory input from $M$ cones and inhibitory inputs from L cones, the red off-centre cell being more strongly inhibited than the green on-centre cell. In this stimulus situation, with stimuli being darker than the adaptation field, PCL off-centre cells are the most sensitive.

the receptive fields of cells are stimulated by successively lower and higher luminances than that of some mean adaptation level. This situation can to some extent be simulated experimentally by alternating light stimuli of different luminances with an adaptation stimulus of constant luminance, a situation obviously different from when stimuli are briefly superimposed on a background. If experimentally, a stimulus replaces a white adaptation field, the ratio $Y$ of stimulus luminance to adaptation field luminance corresponds to luminance factor, a coordinate often used in the representation of surface colours in colorimetry and colour systems, the two other dimensions being, for instance, opponent chrominances $[13,14,17]$.

When light spots are projected upon a steady white background, among opponent PCL cells, on-centre cells are the mot sensitive. Red and green off-centre cells are so strongly inhibited by the white background stimulus [17] that a much stronger excitatory stimulus is needed to evoke an on-response. Under successive contrast conditions, as in the natural daylight environment where surfaces of most objects reflect less light than a white, diffusely reflecting surface, off-centre cells become more sensitive with strong excitatory responses to dark colours as shown in Fig. 6.

3.2.1. Neuronal coding of object colours. In these experiments, we used a similar procedure to that for a steady background, except that the stimuli $\left(4^{\circ} \times 5^{\circ}\right)$ were alternated with a white adaptation field, of $110 \mathrm{~cd} / \mathrm{m}^{2}(3100 \mathrm{td})$. Cell response was plotted as a function of luminance ratio ( $Y=L / L_{\mathrm{a}}$ ), the ratio of stimulus luminance, $L$, to adaptation field luminance, $L_{\mathrm{a}}$. Figure 6 shows such plots for two different cells; a red off-centre cell and a green on-centre cell. A luminance ratio less than one implies a colour darker than the white adaptation field, as with a passively reflecting surface of a coloured object.

It is apparent in Fig. 6 that for a M-L combination of cone input, the off-centre cell, for which the inhibitory L-cone mechanism is relatively strong, is the more sensitive to dark colours with a low luminance ratio threshold of between 0.01 and 0.001 , whereas the threshold of the weakly inhibited

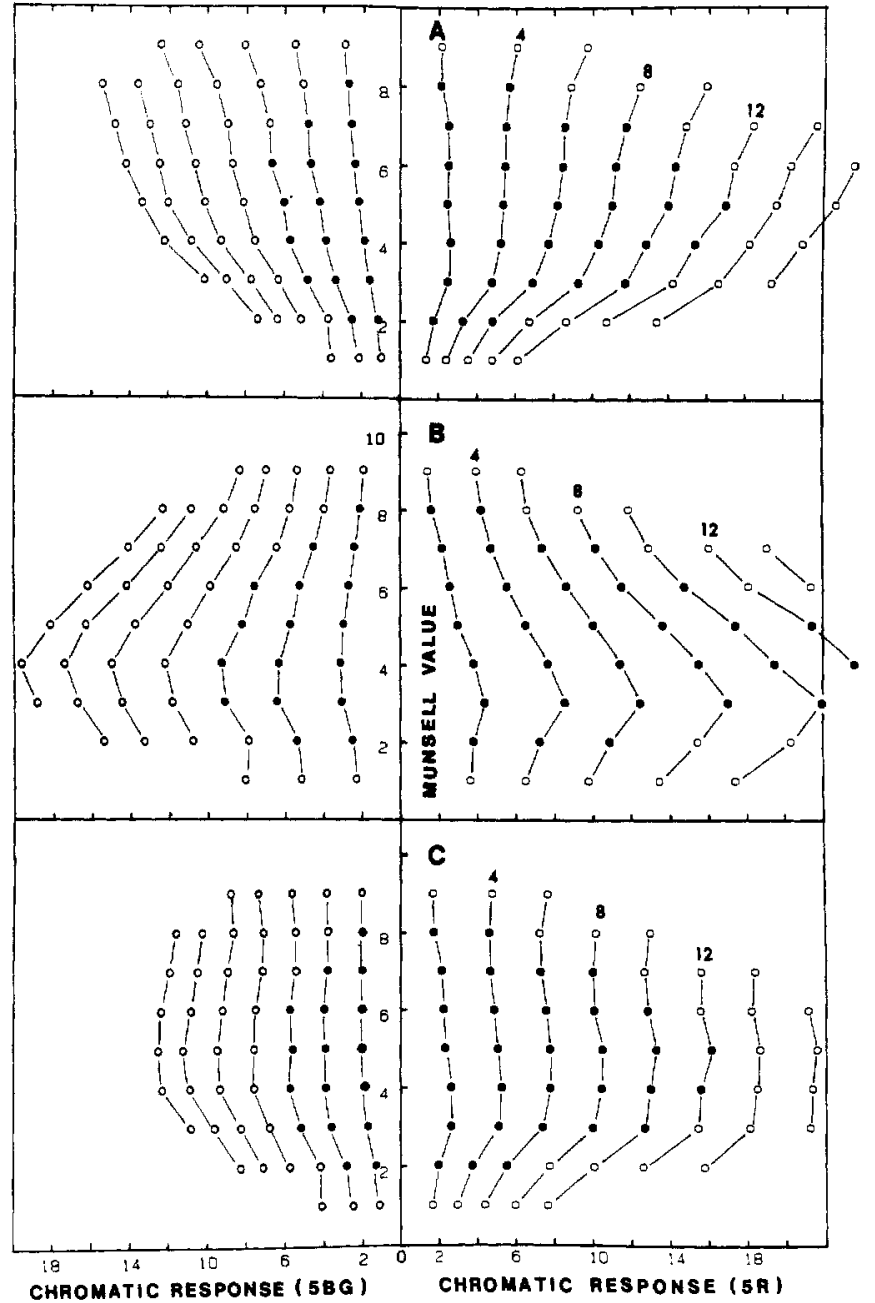

Fig. 7. Computed responses of a sum of on- (A) and off-centre (B) PCL cells with $\mathrm{M}-\mathrm{L}$ and $\mathrm{L}-\mathrm{M}$ cone inputs to the colours of the Munsell system (Chroma 2 to 14 of hues 5R and 5BG). The combined on- and off-cell output is shown in $(\mathrm{C})$.

on-centre cell, is typically about $1 \mathrm{log}$ unit higher. However, there is a wide distribution of sensitivity and responsiveness among both on- and off-centre cells with the effect to increase bandwidth in terms of luminance ratio for groups of cells relative to that of individual cells. This also applies to cell types with the opposite combination of cone input $(\mathrm{L}-\mathrm{M})$, the green off-centre and red on-centre cells, respectively.

These results, with on-centre cells being more responsive for light colours and off-centre cells more responsive for dark colours, suggest that both groups participate in the coding of colour information, but with different ranges of luminance ratio. It is possible [13], by adding the outputs of stronglyand weakly inhibited cells with the same cone combination, to account for suprathreshold scaling of colour differences, as found in the Optical Society of America's Uniform Colour Scale (OSA-UCS) and the Munsell System [25, 26]. The mathematical model of eqs. (1) and (2) permits transformations between the CIE colour coordinates of each sample in these colour systems and the responses of single cells, thus obviating recordings of cell activities to all these colours.

Using this model, we have computed the expected neural outputs of typical cells as functions of the CIE $X, Y$, and $Z$ tristimulus values of OSA-UCS $[25,26]$ and Munsell colour samples [26]. Figure 7 displays the results obtained when computing the responses of weakly and strongly opponent cells 
separately and in combination. Fig. 7A shows the chromatic response of the sum of two weakly inhibited, on-centre cells with opposite $\mathrm{M}-\mathrm{L}$ and $\mathrm{L}-\mathrm{M}$ cone inputs, to Munsell Chroma ranging from 2 to 14 of hues $5 \mathrm{R}$ and 5BG. The computation is done separately for each lightness value (Value 1 to 9). Filled circles represent samples actually used in psychophysical scaling experiments, while open circles are extrapolated values [27]. Circles connected with lines represent constant Munsell Chroma. Both cells responded to stimuli, complementary in colour to their preferred hue direction, and in these theoretical plots we assume a relatively high firing rate to achromatic lights, such that the cells respond to the full range of complementary stimuli shown here (i.e., complete suppression of firing of the red on-centre cell occurs above Chroma 14 for hue 5BG).

Figure $7 \mathrm{~B}$ shows the computed chromatic response sum of two strongly inhibited off-cells with the same cone inputs as in Fig. 7A, and likewise with a cut off (zero imp/s) outside the Chroma range shown here. Because on-centre cells with weak inhibition are more responsive to brighter colours, this leads to constant Chroma lines tilted away from the achromatic axis with increasing luminance ratio (increasing Value), while off-cells are more responsive to darker colours, giving Chroma lines tilted in the other direction. Because of this bias in opposite directions for the different cell types, the combined response of on- and off-centre cells, shown in Fig. 7C. yields Chroma lines parallel to the achromatic axis. The only exception is for the darkest (extrapolated) colours of Values 1 and 2, where the summed chromatic responses are too small. The result for dark colours would have improved if we had used response characteristica of groups of cells in the model instead of typical individual cells. Results similar to those of Fig. 7 were obtained for Value-Chroma planes of other hues, thus confirming that, along the reddish-greenish dimension of colour space, both weakly- and strongly inhibited LGN cells contribute to colour scaling.

The Chroma spacing is smaller for the hue 5BG than for $5 R$. In order to obtain a uniform colour space throughout, with the same distance approximating the same sensory colour difference, a second state opponent transformation of PCL outputs seems necessary [13]. It is possible, by a linear transformation of LGN cell output, to make constant Chroma loci approximate concentric circles about the achromatic point:

$$
\begin{aligned}
F_{1} & =1.5 P_{1}-0.5 P_{2} \\
F_{2} & =0.7 P_{2} \\
F & =\left(F_{1}^{2}+F_{2}^{2}\right)^{1 / 2}
\end{aligned}
$$

$P_{1}$ and $P_{2}$ are the combined responses of PCL on- and offcentre cells [13]. $F$ corresponds to Chroma, and different hues are, at this second, cell opponent stage, coded by the relative activities $F_{1}$ and $F_{2}$ of several LGN cell groups, by assuming vector addition of four orthogonal processes [13]. In this scheme, constant hue curves of the Munsell System approximate straight lines radiating from the achromatic point. The change in hue with changing purity for equiluminous stimuli of constant dominant wavelength (Abney effect [28]) is accounted for by a change in the relative activity $\left|F_{1}\right| /\left|F_{2}\right|$ of the non-linear cell responses to such stimuli. Even if the simple transformation of eq. (3) is only an approximation to a uniform colour space (no attempt of optimisation has been made), it is probably as good, if not better than the colour difference equations recommended by the CIE [13].

It has been pointed out elsewhere [17], that the isolation of responses to chromatic stimuli and ellimination of responses to achromatic stimuli is possible, theoretically, by subtracting outputs of cells with the same receptive field size and retinal location, but with opposite cone inputs (i.e., of red and green on-centre cells, and of red and green off-centre cells). The coding of the third dimension, lightness (for objects) or brightness (for light sources), may be solved in a similar way [17, 29]. An intensity code could be provided by adding the activites of on-centre cells (for instance red and green on-centre cells) to yield a "brightness" signal, and of off-centre cells to provide a signal for "blackness" of surface colours.

3.2.2. Changes of hue and saturation with luminance ratio. It is a common observation that when the luminance ratio of a stimulus of constant chromaticity is increased from zero, the perceived saturation (or colour strength) increases to a maximum and then decreases again, much in the same way as the cell responses of Fig. 6 change with luminance ratio. This change is accompanied by a change in hue where, for instance, long-wavelength lights become more and more yellowish as luminance ratio increases (Bezold-Bruecke effect $[30,31])$. Whereas the hue change has been explained by the non-linear responses of cones themselves [32], the saturation change is more readily accounted for by cone-opponency and the resulting non-monotonic response behaviour of cells. On the assumption that saturation and hue percepts are both related to the relative activities of opponent cells, one may ask if the same second stage transformation $\left(F_{1}, F_{2}\right)$ of eq. (3) that accounts so well for scaling also predicts the dependence of these attributes on intensity.

Figure 8A shows the $\left(F_{1}, F_{2}\right)$ Chroma-coordinates predicted by this second stage opponent transformation for some selected wavelengths when luminance ratio increases over a $4 \mathrm{log}$ unit range from about 0.003 for black to about 30 . The radial distance $F$ from origo in any direction is proportional to Munsell Chroma, and the angle of this radius vector relative to the coordinate axes represents hue. Arrows indicate similar luminance ratios in $A$ and $B$. It may seem that the changes of hue and colour strength predicted by this plot are much too large, especially at the blue and red ends of the spectrum. However, when these results are compared with subjective magnitude estimations of colour strength and hue, such as those plotted in Fig. 8B for one observer, they are similar to the subjective ratings. This indicates that the nonlinear response behaviour of LGN opponent cells may be a common source for both the observed Bezold-Bruecke hue shift and the non-monotonic dependency of colour strength on luminance ratio. The model response $F$ of Fig. 8A also gives some indication that 80 units is about the maximum Chroma sensation that can be evoked by these interference filters.

\section{Discussion}

We have attempted here to indicate some ways in which neurophysiological data my be related to psychophysical results. The results suggest that different classes of visual neurones may be involved in detection and discrimination of colour stimuli depending on the stimulus conditions. For example, with a steady white background, detection can be 


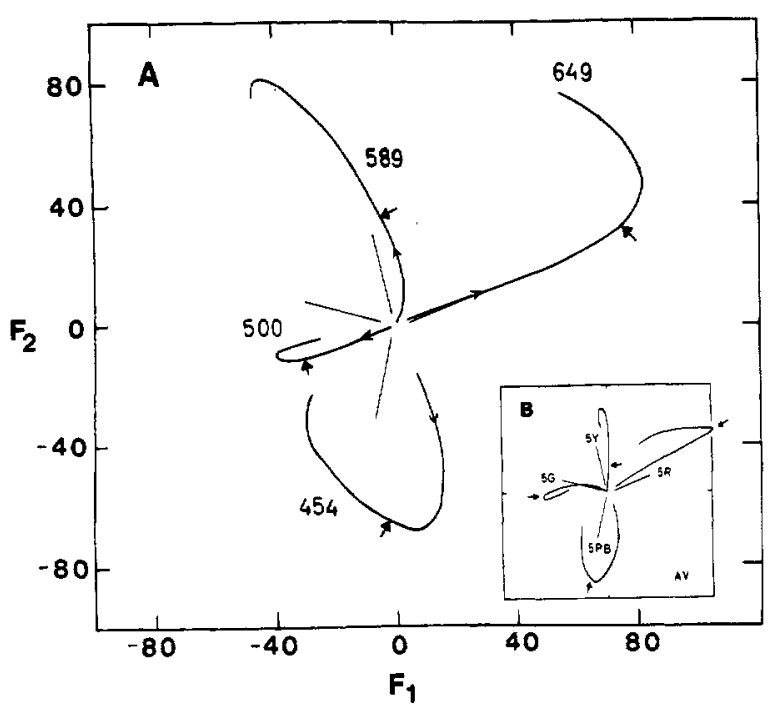

Fig. 8. (A) shows the model responses $F_{1}$ and $F_{2}$ at a hypothetical "cell opponent stage" (a linear combination of outputs of PCL cells of the LGN) to four different chromatic stimuli of the dominant wavelengths indicated when they increase in luminance ratio relative to the adaptation field. In the inset (B), psychophysical scaling of relative chromatic strength (distance from the neutral point) and hue angle are shown for the same stimuli. The psychophysical ratings are normalised with respect to elementary hues corresponding to Munsell hues $5 \mathrm{R}, 5 \mathrm{Y}, 5 \mathrm{G}$ and $5 \mathrm{~PB}$. Arrows indicate the same luminance ratio $Y$ of stimulus to adaptation field in (A) and (B). Both plots show that chromatic strength $F$ increases with luminance ratio to reach a maximum and then decreases again. This saturation change is accompanied by a hue shift towards yellow for long wavelength lights and towards bluegreen for short wavelengths which is also well predicted by the opponent model in (A) (except for the hue shift of the $500 \mathrm{~nm}$ stimulus).

achieved either through MCL, non-opponent cells or through PCL opponent cells, depending on which class is the most sensitive for a given stimulus. Chromatic discrimination is also a threshold task, and is mediated by opponent, usually on-centre cells. Off-centre opponent cells are too insensitive in this stimulus situation to play a role near threshold.

The notion that the most sensitive neurones are responsible for detection and discrimination seems reasonable near threshold, but under suprathreshold conditions interactions and combinations of cell outputs are likely to be responsible for the percept. For example, in a more natural viewing condition with stimuli both brighter and darker than some mean illumination level, PCL off-centre cells have the highest sensitivity to dark colours (Fig. 6), but they do not respond well to stimuli brighter than the adaptation level, when PCL on-centre cells are most active. Thus on- and off-centre systems share the task of representing the entire scale of luminance ratios, on-centre cells being best suited for coding of the brighter colours of selfluminous objects, and off-centre cells for coding the colours of darker, passively reflecting surfaces.

It is possible to analyse this coding through combination of cell outputs in a formal manner. We have demonstrated that sums of responses of on- and off-centre cells with the same cone inputs (either $\mathrm{M}-\mathrm{L}$ or $\mathrm{L}-\mathrm{M}$ ), and a subsequent vector addition of outputs of cells with different cone input combinations can account well for colour scaling, so that constant ratios of activities of groups of cells may correlate with constant perceived hue, and constant response differences with equal chromatic differences [13]. An additional advantage of summing the outputs of on- and off-centre cells with the same cone combination has been pointed out by D'Zemura and Lennie [33]; a cell receiving such inputs would be less sensitive to changes in the spatial properties of a stimulus, and thus less ambiguous in its colour code.

Although psychophysical performance is constrained in the first instance by the receptors, we must also consider the many different transformations of the same cone signals in different types of neurones. Indeed, several recent studies conclude that an opponent combination of cone signals results in a more efficient transfer of colour information [34], and that chromatic thresholds may require less modulation of cone outputs than pure intensity thresholds [35]. The failure of trichromatic theory to adequately account for such data suggests that a study of the diversity of cone pathways and consequent parallel processing of optical information may be the best way to understand visual performance. By opening the "Black Box" and studying the neuronal systems present, we hope to be able to develop more realistic models of colour vision.

\section{Acknowledgment}

We would like to thank Prof. O. D. Creutzfeldt for his generous support and D. Tigwell, J. Crook, T. Seim, and J. Tryti for valuable asistance and discussion. This work was partly supported by NATO collaboration Research Grant 650/83 and the Norwegian Research Council for Science and the Humanities.

\section{References}

1. Schroedinger, E., Ann. Physik 63, 481 (1920).

2. Helmholtz, H. von, Handbuch der Physiologischen Optik, p. 447, Hamburg, 1896.

3. Stiles, W. S., Proc. Phys. Soc. (London) 58, 41 (1946).

4. Nunn, B. J., Schnapf, J. L. and Baylor, D. A., Nature 309, 264 (1984).

5. Schnapf, J. L., Kraft, T. W. and Baylor, D. A., Nature 325, 439 (1987).

6. De Valois, R. L., Morgan, H. C., Polson, M. C., Mead, W. R. and Hull, E. M., Vision Res. 14, 53 (1974).

7. de Monasterio, F. M. and Gouras, P., J. Physiol. 251, 167 (1975).

8. de Monasterio, F. M., J. Neurophysiol. 41, 1394 (1976).

9. Wiesel, T. N. and Hubel, D. H., J. Neurophysiol. 29, 1115 (1966).

10. Dreher, B., Fukuda, Y. and Rodieck, R. W., J. Physiol. 258, 433 (1976).

11. Creutzfeldt, O. D., Lee, B. B. and Elepfandt, A., Expl. Brain. Res. 35, 527 (1979)

12. Perry, V. H., Oehler, R. and Cowey, A., Neuroscience 12, 1110 (1984).

13. Valberg, A., Seim, T., Lee, B. B. and Tryti, J., J. Opt. Soc. Amer. 3, 1726 (1986).

14. Lee, B. B., Valberg, A., Tigwell, D. A. and Tryti, J., Proc. Roy. Soc. B230, 293 (1987).

15. Valberg, A., Lee, B. B., Tigwell, D. A. and Creutzfeldt, O. D., Expl. Brain Res. 58, 604 (1985).

16. Valberg, A., Lee, B. B. and Tigwell, D. A., Vision Res. 26, $106 \mathrm{l}$ (1986).

17. Valberg, A., Lee, B. B. and Tryti, J., Vision Res. 27, 867 (1987).

18. Crook, J. M., Lee, B. B., Tigwell, D. A. and Valberg, A., J. Physiol. London 392, 193 (1987).

19. Sperling, H. G. and Harwerth, R. S., Science 172, 180 (1971).

20. King-Smith, P. E. and Carden, D., J. Opt. Soc. Amer. 66, 709 (1976).

21. de Monasterio, F. M. and Schein, S. J., J. Physiol. London 299, 385 (1980).

22. Wyszecki, G. and Stiles, W. S., Color Science: Concepts and Methods, Qantitative Data and Formulae, Table 3, p. 618. New York, 1982.

23. Abney, W. de W., Researches in Color Vision and Trichromatic Theory, p. 418. London (1913).

24. Graham, C. H. and Hsia, Y., J. Opt. Soc. Amer. 59, 993 (1969).

25. MacAdam, D. L., J. Opt. Soc. Amer. 68, 121 (1978).

26. Wyszecki, G. and Stiles, W. S., Color Science: Concepts and Methods, Quantitative Data and Formulae, New York (1982).

27. Newhall, S. M., J. Opt. Soc. Amer. 30, 617 (1940).

28. Abney, W. de W., Proc. Roy. Soc., London A23, 120 (1910). 
29. Creutzfeldt, O., Lee, B. B. and Valberg, A., Exp. Brain Res. 63, 21 (1986).

30. Purdy, M. D., Am. J. Psychol. 43, 541 (1931).

31. Purdy, M. D., Am. J. Psychol. 49, 313 (1937).
32. Vos, J. J., Vision Res. 26, 337 (1986).

33. D'Zemura, M. and Lennie, P., J. Opt. Soc. Amer. 3, 1662 (1986).

34. Buchsbaum, G. and Gottschalk, A., Proc. Roy. Soc. B220, 89 (1983).

35. Mullen, K. T., J. Physiol. 359, 381 (1985). 